\title{
1.9-kV AlGaN/GaN Lateral Schottky Barrier Diodes on Silicon
}

\author{
Mingda Zhu, Bo Song, Meng Qi, Zongyang Hu, Kazuki Nomoto, Xiaodong Yan, Yu Cao, Member, IEEE, \\ Wayne Johnson, Erhard Kohn, Debdeep Jena, Senior Member, IEEE, and \\ Huili Grace Xing, Senior Member, IEEE
}

\begin{abstract}
In this letter, we present AlGaN/GaN lateral Schottky barrier diodes on silicon with recessed anodes and dual field plates. A low specific ON-resistance $R_{\mathrm{ON}, S P}\left(5.12 \mathrm{~m} \Omega \cdot \mathrm{cm}^{2}\right)$, a low turn-ON voltage $(<0.7 \mathrm{~V})$, and a high reverse breakdown voltage $(\mathrm{BV})(>1.9 \mathrm{kV})$ were simultaneously achieved in devices with a $25-\mu \mathrm{m}$ anode/cathode distance, resulting in a power figureof-merit $\mathrm{BV}^{2} / R_{\mathrm{ON}}, \mathrm{SP}$ of $727 \mathrm{MW} \cdot \mathrm{cm}^{-2}$. The record high $\mathrm{BV}$ of $1.9 \mathrm{kV}$ is attributed to the dual field-plate structure.
\end{abstract}

Index Terms-Schottky barrier diode, GaN on silicon, breakdown voltage, high voltage device, AIGaN/GaN, field plate.

\section{INTRODUCTION}

G aN ON silicon technology has attracted tremendous research attention for its potential to be widely commercialized thanks to its competitive performance and low cost. Diode based rectifiers, as an indispensable device for most power electronic applications, require low turn-on voltage and specific on resistance $\left(R_{O N, S P}\right)$, low reverse leakage current yet high breakdown voltage (BV) to minimize power loss during operation. AlGaN/GaN based Schottky barrier diodes (SBDs) on sapphire or SiC substrate have shown superior performance over rectifiers achieved in other material systems due to the wide band gap of $\mathrm{GaN}$ and high electron mobility in the two dimensional electron gas (2DEG). However, the performance of $\mathrm{GaN}$ on silicon rectifier has been lagging behind.

Recessed anode was reported effective in reducing the turn-on voltage hence specific on-resistance of SBDs [1]. Meanwhile, field plate technology was proven effective in boosting the breakdown voltage in both field effect transistors (FETs) [2] and SBDs [3]. The combination of recessed anode and field plate has been implemented in AlGaN/GaN SBDs with excellent power figure-of-merit (FOM) $\mathrm{BV}^{2} / R_{O N, S P}[1]$. To further improve the breakdown voltage, we have adopted a

Manuscript received December 5, 2014; revised January 6, 2015 and January 25, 2015; accepted February 12, 2015. Date of publication February 16, 2015; date of current version March 20, 2015. This work was supported in part by the DARPA MPC and ARPAe SWITCHES projects. The review of this letter was arranged by Editor D.-H. Kim.

M. Zhu, M. Qi, Z. Hu, K. Nomoto, X. Yan, and E. Kohn are with the Department of Electrical Engineering, University of Notre Dame, Notre Dame, IN 46556 USA (e-mail: mzhu3@nd.edu).

B. Song, D. Jena, and H. G. Xing are with the Department of Electrical Engineering, University of Notre Dame, Notre Dame, IN 46556 USA, and also with Cornell University, Ithaca, NY 14850 USA (e-mail: grace.xing@cornell.edu).

Y. Cao was with IQE LLC, Westborough, MA 01581 USA. He is now with HRL Laboratories, LLC, Malibu, CA 90265 USA.

W. Johnson is with IQE LLC, Westborough, MA 01581 USA.

Color versions of one or more of the figures in this letter are available online at http://ieeexplore.ieee.org.

Digital Object Identifier 10.1109/LED.2015.2404309

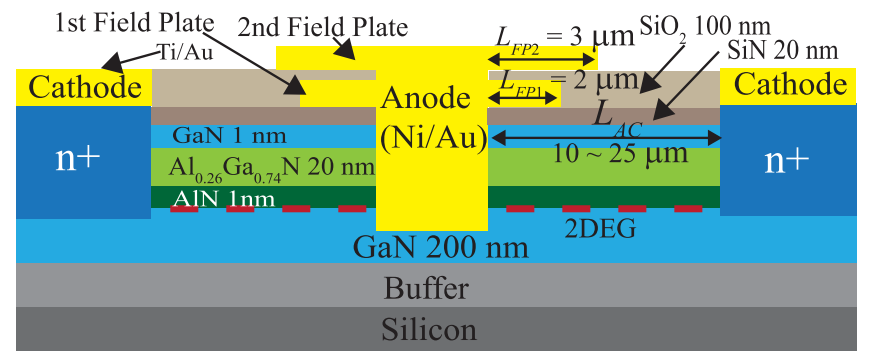

Fig. 1. Schematic cross section of the recessed anode Schottky barrier diode with double field plates.

dual field plate structure with the recessed anode in this letter. $\mathrm{SiN}_{\mathrm{x}}$ and $\mathrm{SiO}_{2}$ by atomic layer deposition (ALD) were used as dielectric for the first and second field plate, respectively. Fabricated devices show a maximum breakdown voltage of $1.93 \mathrm{kV}$ with a power FOM of $727 \mathrm{MWcm}^{-2}$, and this $\mathrm{BV}$ value is the highest among the reported GaN-on-Si diodes.

\section{FABRICATION PROCESS}

The epitaxial layers were grown by metal organic chemical vapor deposition on 6 inch silicon substrates, consisting of $1 \mathrm{~nm} \mathrm{GaN}, 20 \mathrm{~nm} \mathrm{Al} 0.26 \mathrm{Ga}_{0.74} \mathrm{~N}, 1 \mathrm{~nm} \mathrm{AlN,} 200 \mathrm{~nm} \mathrm{GaN}$, and $4 \mu \mathrm{m}$ buffer layer. All the epitaxial layers were grown along the metal face crystal orientation, i.e. [001]. Fabrication of the SBDs started with the cathode ohmic contacts using the molecular beam epitaxy regrowth [4] and non-alloyed $\mathrm{Ti} / \mathrm{Au}$ metallization. The regrown $\mathrm{GaN}$ has a thickness of $100 \mathrm{~nm}$ and a Si doping concentration of $1 \times 10^{20} \mathrm{~cm}^{-3}$. ALD SiN of $20 \mathrm{~nm}$ thick was then deposited as dielectric for the first field plate, which was chosen so that the pinchoff voltage of the resultant metal-SiN-HEMT structure is no less than $-10 \mathrm{~V}$. The anode was defined by optical lithography and etching to remove $\mathrm{SiN}$ and $50 \mathrm{~nm}$ of $\mathrm{AlGaN} / \mathrm{GaN}$ using $\mathrm{CF}_{4}$ and $\mathrm{BCl}_{3}$ plasma, respectively. The anode contact and the first field plate with a length $\left(L_{F P 1}\right)$ of $2 \mu \mathrm{m}$ was then defined by $\mathrm{Ni} / \mathrm{Au}$ deposition and liftoff. $\mathrm{ALD} \mathrm{SiO}_{2}$ of $100 \mathrm{~nm}$ was subsequently deposited, followed by the definition of the second field plate with a length $\left(L_{F P 2}\right)$ of $3 \mu \mathrm{m}$. A relatively thick $\mathrm{SiO}_{2}$ was used to support the large voltage drop across the anode and cathode. The Hall effect measurement on the fabricated devices shows a 2DEG concentration of $9.8 \times 10^{12} \mathrm{~cm}^{-2}$ with a mobility $\mu$ of $1490 \mathrm{~cm}^{2} / \mathrm{V} \cdot \mathrm{s}$. The anode/cathode distance $\left(L_{A C}\right)$ was varied between 10 and $25 \mu \mathrm{m}$. The cross-sectional schematic of a finished device is shown in Fig. 1. 

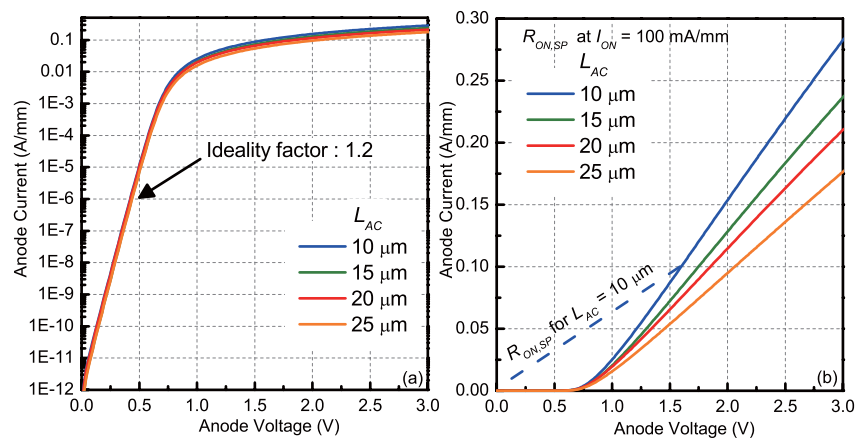

Fig. 2. Forward bias $I-V$ characteristics in (a) semi-log and (b) linear scale of the fabricated GaN-on-Si SBDs with recessed anode and double field plates. The dash line in (b) illustrates the definition of $R_{O N, S P}$ used in this letter.

\section{EXPERIMENTAL RESULTS}

Figure 2 shows the typical forward bias characteristics of the fabricated diodes with various anode/cathode distances $\left(L_{A C} \sim 10-25 \mu \mathrm{m}\right)$. The ideality factor and the turn-on voltage $V_{\text {turn-on }}$ (at $1 \mathrm{~mA} / \mathrm{mm}$ ) of the SBDs are $1.22 \pm 0.05$ and $0.67 \pm 0.02 \mathrm{~V}$ across the sample. Similar $V_{\text {turn-on }}$ values have been reported previously in recessed anode GaN SBDs where the anode metal is in direct contact with the 2DEG at the $\mathrm{AlGaN} / \mathrm{GaN}$ interface [1], [5]. It is worth noting that overlapping current - voltage characteristics over 9 orders of magnitude in the linear regime of the semi-log plot shown in Fig. 2(a) are observed. Since the forward current for $V<V_{\text {turn-on }}$ is dominated by the Schottky junction not by the series resistance, this, along with the small variations of the aforementioned ideality factor and $V_{t u r n-o n}$, shows excellent Schottky junction uniformity among different devices, regardless of the $L_{A C}$.

The reverse bias characteristics of the same diodes shown in Fig. 2 are plotted in Fig. 3 (a). All devices show a low reverse leakage current $(<5 \mu \mathrm{A} / \mathrm{mm})$ up to $700 \mathrm{~V}$. With an increasing $L_{A C}$, a larger breakdown voltage was obtained, which leads to the well-known tradeoff between breakdown voltage and on-resistance. A breakdown voltage (measured at $1 \mathrm{~mA} / \mathrm{mm}$ ) of $1.93 \mathrm{kV}$ has been achieved with a $L_{A C}$ of $25 \mu \mathrm{m}$ and a $R_{O N, S P}$ of $5.12 \mathrm{~m} \Omega \cdot \mathrm{cm}^{2}$ (extracted at a forward current of $100 \mathrm{~mA} / \mathrm{mm}$, as illustrated in Fig. 2(b)), resulting in a power FOM of $727 \mathrm{MW} / \mathrm{cm}^{2}$. The device with a $L_{A C}$ of $20 \mu \mathrm{m}$ shows a breakdown voltage of $1.6 \mathrm{kV}$ and a $R_{O N, S P}$ of $3.7 \mathrm{~m} \Omega \cdot \mathrm{cm}^{2}$, resulting in a power FOM of $691 \mathrm{MW} / \mathrm{cm}^{2}$. The $\mathrm{BV}$ of $1.93 \mathrm{kV}$ is the highest value among the reported $\mathrm{GaN}$ on $\mathrm{Si}$ diodes while both FOM values are comparable to the other state-of-the-art devices. The capacitance versus voltage $(C-V)$ measurement and zoomed in reverse $I-V$ results are plotted in the inset of Fig. 3(a), showing at a reverse bias of $7 \mathrm{~V}$ a complete depletion of the 2DEG underneath the field plates ( $V_{\text {pinchoff }} \sim-7 \mathrm{~V}$ ) and a simultaneous saturation of reverse leakage current, indicating the effect of the field plates in curbing the leakage current. The device switching time can also be estimated based on the $C-V$ and $I-V$ characteristics as follows: $\tau_{\text {switch }} \sim C_{\text {max }} \bullet V_{\text {pinchoff }} / I_{\text {on }} \sim 350 \mathrm{ps, \text {indicating }}$ these devices are fast even though the double FPs increase the device capacitance considerably compared to devices without FPs.
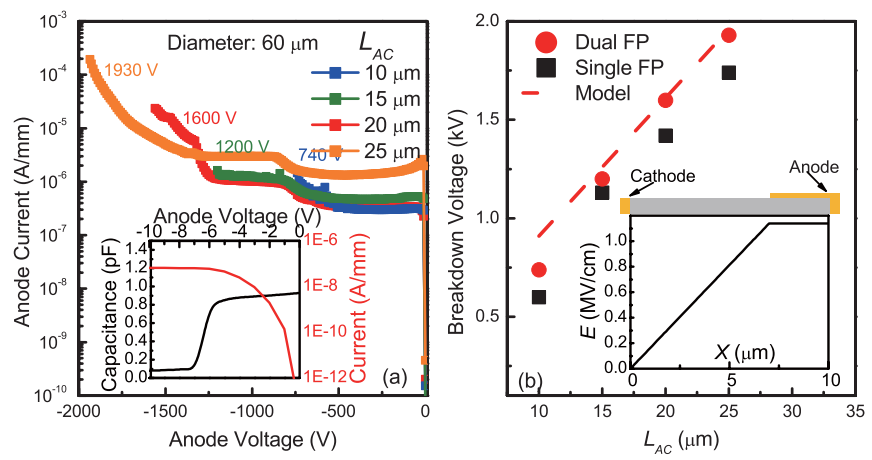

Fig. 3. (a) Reverse bias $I-V$ characteristics of the diodes shown in Fig. 2. Inset: diode $C-V$ at $1 \mathrm{MHz}$ and reverse bias $I-V$ up to $-10 \mathrm{~V}$. (b) $\mathrm{BV}$ versus $L_{A C}$ for SBDs with double and single field plate structures. The dash line is the calculated BV using an $E_{C r}$ of $1.4 \mathrm{MV} / \mathrm{cm}$ and the trapezoid file profile shown in the inset.

The record high breakdown voltage is attributed to the double field plate structure, which is often used in power FETs to reduce peak electric field thus increasing BV with the same voltage bearing distance. To investigate the effect of the second field plate, another set of devices were fabricated with the first field plate only and an otherwise identical process flow. The plot of BV versus $L_{A C}$ for the diodes with the single and double field plate structures is shown in Fig. 3(b). A noticeable improvement of BV $(\sim 5-25 \%)$ is observed for each $\mathrm{L}_{A C}$ with the addition of the second field plate. A nearly linear increase of breakdown voltage is also observed with increasing $L_{A C}$, indicating depletion of the 2DEG beyond the field plate region. Simulations show the electric field typically spikes near the FP edges on the drain side, thus difficult to analytically extract the breakdown field in lateral power devices. To gauge the efficacy of the double FP structure, we compute an effective breakdown field by assuming a total depletion of the 2DEG between the anode and cathode and a constant lateral electric field underneath the field plates. The resulting field profile is trapezoidal as shown in the inset of Fig. 3(b), which also represents an optimized field profile. The effective critical electric field ( $\left.E_{c r, e f f}\right)$ is then calculated to be 1.14-1.4 MV/cm, which is much lower than the value in bulk $\mathrm{GaN}$ (3.4 MV/cm [6]) due to the aforementioned challenges in engineering the surface field in these diodes. This low $E_{c r, e f f}$ also indicates that there is still room to improve the field profile thus device BV. The observation of a nearly linear increase in $\mathrm{BV}$ versus $L_{A C}$, also reported by other groups [1], [5], is worthy further investigation.

Figure 4 presents the benchmark plot of $\mathrm{BV}$ versus $R_{O N, S P}$ for the $\mathrm{GaN}$ based unipolar power diodes, including both lateral and vertical devices. The SBDs with recessed anodes in this letter are among the best reported and show the highest $\mathrm{BV}$ among $\mathrm{GaN}$ power diodes on silicon substrates, and compare favorably to the state-of-the-art $\mathrm{GaN}$ diodes on $\mathrm{SiC}$, sapphire and bulk $\mathrm{GaN}$ substrates that often has less number of dislocations than GaN-on-Si. In comparison to the previously reported SBDs with recessed anodes in direct contact to the 2DEG, the devices in this letter show $\sim 2 \mathrm{X}$ improvement in BV: $1900 \mathrm{~V}$ vs. $800 \mathrm{~V}$ for GaN-on-Si SBDs [5] and $1900 \mathrm{~V}$ vs. $1000 \mathrm{~V}$ for GaN-on-SiC SBDs [1]. The $R_{O N, S P}-\mathrm{BV}$ values of all these $\mathrm{SBDs}$ with recessed anode 


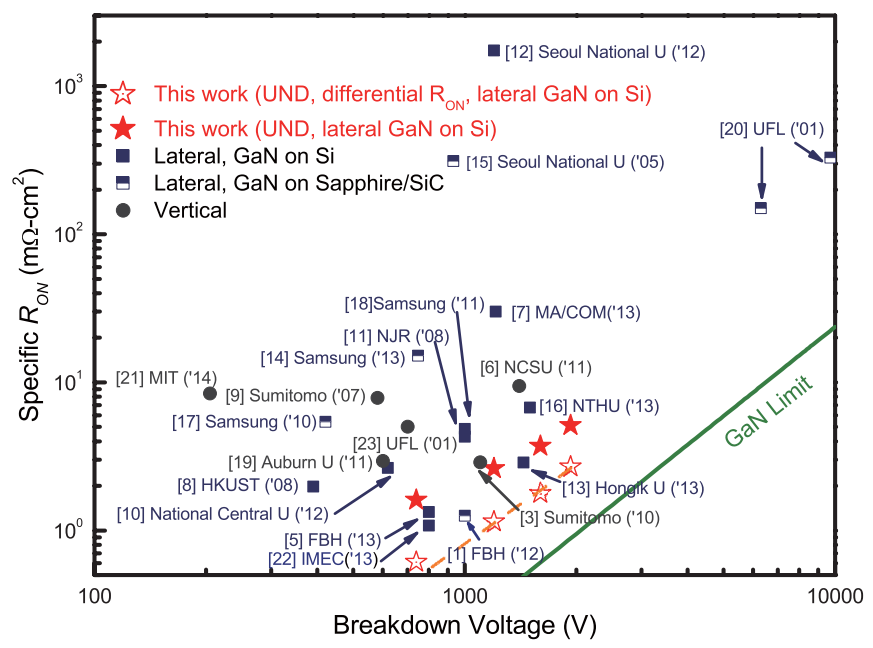

Fig. 4. The benchmark plot of BV versus $R_{O N, S P}$. All the reference values are re-calculated based on the reported data following the definition of $R_{O N, S P}$ shown in Fig. 2(b) so that the diode turn-on effect is also taken into account, using $I_{O N}=100 \mathrm{~mA} / \mathrm{mm}$ criteria. The differential $R_{O N}$ of the diodes in this letter is also extracted (hollow stars) and modeled (dash line) assuming $\mu=1490 \mathrm{~cm}^{2} / \mathrm{V} \cdot \mathrm{s}$ and $E_{C r}=1.5 \mathrm{MV} / \mathrm{cm}$. The green line shows the FOM limit of bulk GaN using $\mu=500 \mathrm{~cm}^{2} / \mathrm{V} \cdot \mathrm{s}$ and $E_{C r}=3.4 \mathrm{MV} / \mathrm{cm}$. UND stands for the University of Notre Dame.

fall along the same FOM line representing the best achieved SBDs experimentally (Fig. 4), which suggests the recess anode coupled with multiple FPs is a viable technology to achieve high performance $\mathrm{GaN}$ rectifiers. Given the advantage in terms of cost GaN-on-Si technology has over other substrates, the SBDs with recessed anode and double field plate structure has great potential for power electronic applications, which are highly sensitive to cost due to the continuous performance improvement in silicon based power devices.

\section{CONCLUSION}

Employing a recessed anode and double field plate structure, we demonstrated $\mathrm{AlGaN} / \mathrm{GaN}$-on-Si SBDs, exhibiting a record breakdown voltage of $1.93 \mathrm{kV}$ and competitive power FOM values. The high performance, together with the low cost associated with the GaN-on-Si technology, shows a great potential for its applications in power electronics.

\section{REFERENCES}

[1] E. Bahat-Treidel et al., "Fast-switching GaN-based lateral power Schottky barrier diodes with low onset voltage and strong reverse blocking," IEEE Electron Device Lett., vol. 33, no. 3, pp. 357-359, Mar. 2012.

[2] H. Xing et al., "High breakdown voltage AlGaN-GaN HEMTs achieved by multiple field plates," IEEE Electron Device Lett., vol. 25, no. 4, pp. 161-163, Apr. 2004.
[3] Y. Saitoh et al., "Extremely low on-resistance and high breakdown voltage observed in vertical GaN Schottky barrier diodes with high-mobility drift layers on low-dislocation-density GaN substrates," Appl. Phys. Exp., vol. 3, no. 8, pp. 081001-1-081001-3, Jul. 2010.

[4] J. Guo et al., "Metal-face InAlN/AlN/GaN high electron mobility transistors with regrown ohmic contacts by molecular beam epitaxy," Phys. Status Solidi A, vol. 208, no. 7, pp. 1617-1619, Jul. 2011.

[5] E. B. Treidel et al., "Fast GaN based Schottky diodes on Si(111) substrate with low onset voltage and strong reverse blocking," Phys. Status Solidi C, vol. 10, no. 5, pp. 849-852, May 2013.

[6] A. M. Ozbek and B. J. Baliga, "Planar nearly ideal edge-termination technique for GaN devices," IEEE Electron Device Lett., vol. 32, no. 3, pp. 300-302, Mar. 2011.

[7] T. Boles et al., "> 1200 V GaN-on-silicon Schottky diode," Phys. Status Solidi C, vol. 10, no. 5, pp. 835-839, May 2013.

[8] W. Chen et al., "High-performance $\mathrm{AlGaN} / \mathrm{GaN}$ lateral field-effect rectifiers compatible with high electron mobility transistors," Appl. Phys. Lett., vol. 92, no. 25, pp. 253501-1-253501-3, 2008.

[9] S. Hashimoto et al., "High-purity $\mathrm{GaN}$ epitaxial layers for power devices on low-dislocation-density GaN substrates," J. Cryst. Growth, vol. 298, pp. 871-874, Jan. 2007.

[10] Y.-M. Hsin et al., "A $600 \mathrm{~V} \mathrm{AlGaN/GaN} \mathrm{Schottky} \mathrm{barrier} \mathrm{diode} \mathrm{on}$ silicon substrate with fast reverse recovery time," Phys. Status Solidi C, vol. 9, nos. 3-4, pp. 949-952, Mar. 2012.

[11] A. Kamada et al., "High-voltage AlGaN/GaN Schottky barrier diodes on Si substrate with low-temperature GaN cap layer for edge termination," in Proc. 20th Int. Symp. Power Semiconductor Devices IC's, May 2008, pp. $225-228$.

[12] Y. S. Kim et al., "AlGaN/GaN Schottky barrier diode on Si substrate employing $\mathrm{NiO}_{x} / \mathrm{Ni} / \mathrm{Au}$ contact," Jpn. J. Appl. Phys., vol. 51, no. 9S2, pp. 09MC01-1-09MC01-5, 2012

[13] J.-G. Lee et al., "Low turn-on voltage $\mathrm{AlGaN} / \mathrm{GaN}$-on-Si rectifier with gated ohmic anode," IEEE Electron Device Lett., vol. 34, no. 2, pp. 214-216, Feb. 2013.

[14] J.-H. Lee et al., "AlGaN/GaN-based lateral-type Schottky barrier diode with very low reverse recovery charge at high temperature," IEEE Trans. Electron Devices, vol. 60, no. 10, pp. 3032-3039, Oct. 2013.

[15] S.-C. Lee et al., "High breakdown voltage GaN Schottky barrier diode employing floating metal rings on AlGaN/GaN hetero-junction," in Proc. 17th Int. Symp. Power Semiconductor Devices IC's, May 2005, pp. 247-250.

[16] Y.-W. Lian et al., "AlGaN/GaN Schottky barrier diodes on silicon substrates with selective $\mathrm{Si}$ diffusion for low onset voltage and high reverse blocking," IEEE Electron Device Lett., vol. 34, no. 8, pp. 981-983, Aug. 2013.

[17] W. Lim et al., "Temperature dependence of current-voltage characteristics of Ni-AlGaN/GaN Schottky diodes," Appl. Phys. Lett., vol. 97, no. 24, pp. 242103-1-242103-3, 2010.

[18] K. Park et al., " $1 \mathrm{kV}$ AlGaN/GaN power SBDs with reduced on resistances," in Proc. IEEE 23rd Int. Symp. Power Semiconductor Devices IC's, May 2011, pp. 223-226.

[19] Y. Wang et al., "Ultra-low leakage and high breakdown Schottky diodes fabricated on free-standing GaN substrate," Semicond. Sci. Technol., vol. 26, no. 2, p. 022002, Feb. 2011

[20] A. P. Zhang et al., "Lateral $\mathrm{Al}_{x} \mathrm{Ga}_{1-x} N$ power rectifiers with $9.7 \mathrm{kV}$ reverse breakdown voltage," Appl. Phys. Lett., vol. 78, no. 6, pp. 823-825, Feb. 2001.

[21] Y. Zhang et al., "GaN-on-Si vertical Schottky and p-n diodes," IEEE Electron Device Lett., vol. 35, no. 6, pp. 618-620, Jun. 2014.

[22] S. Lenci et al., "Au-free $\mathrm{AlGaN} / \mathrm{GaN}$ power diode on 8-in Si substrate with gated edge termination," IEEE Electron Device Lett., vol. 34, no. 8, pp. 1035-1037, Aug. 2013.

[23] A. P. Zhang et al., "Vertical and lateral $\mathrm{GaN}$ rectifiers on free-standing GaN substrates," Appl. Phys. Lett., vol. 79, no. 10, pp. 1555-1557, 2001. 